\title{
SERUM CARCINOEMBRYONIC ANTIGEN AS AN ADJUNCT TO PREOPERATIVE STAGING OF LUNG CANCER
}

Jeffrey B. Rubins, $\mathrm{MD}^{\mathrm{a}}$

Jordan Dunitz, $\mathrm{MD}^{\mathrm{a}}$

Hanna B. Rubins, MD, MPH ${ }^{\mathrm{b}}$

Michael A. Maddaus, $\mathrm{MD}^{\mathrm{c}}$

Dennis E. Niewoehner, MD

\begin{abstract}
Objective: To determine whether measurement of preoperative serum carcinoembryonic antigen concentrations adds useful prognostic data to current preoperative staging of lung cancer by computed tomography, bronchoscopy, and mediastinoscopy. Methods: A prospective cohort study of 130 consecutive patients was evaluated for suspected lung cancer from July 1991 through December 1992 at a university-affiliated Veterans Affairs Medical Center. Serum concentrations of carcinoembryonic antigen were measured before diagnosis, staging, or resection of cancer. Results: Malignant disease was diagnosed by bronchoscopy, needle biopsy, mediastinoscopy, or resection in 111 of 130 patients. In the 50 patients undergoing resection with curative intent, multivariate analysis indicated that carcinoembryonic antigen was a significant predictor of survival independent of patient age, pathologic stage, histologic type, and tumor size $(P=.0357)$. Conclusions: Elevated preoperative serum concentrations of carcinoembryonic antigen predict a poor prognosis for lung cancer independent of other conventional staging parameters and have an adjunctive role in the staging of lung cancer. (J Thorac Cardiovasc Surg 1998;116:412-6)
\end{abstract}

$A_{0}^{\mathrm{p}}$ proximately $50 \%$ of patients die within 5 years of resection of lung cancer with curative intent, suggesting that the laboratory tests and imaging modalities currently used for preoperative staging have limited sensitivities to detect unfavorable tumor biology and disseminated malignant disease. Although many serologic and tissue tumor markers have been reported to identify unfavorable tumor biology, carcinoembryonic antigen (CEA) is a serum marker that has the potential to augment current staging modalities by identifying advanced lung cancer. ${ }^{1}$ CEAs represent a heterogeneous group of oncofetal glycoprotein antigens that circulate in high concentrations in patients with certain malignant tumors, especially epithelial tumors. Although

From the Pulmonary Disease and General Internal Medicine Divisions, ${ }^{b}$ Department of Medicine, and Department of Thoracic Surgery," Veterans Affairs Medical Center and University of Minnesota School of Medicine, Minneapolis, Minn.

Supported by the Vetcrans Affairs Department of Rescarch.

Received for publication Nov. 24, 1997; revisions requested Jan.

30, 1998; revisions received May 14, 1998; accepted for publication June 3, 1998.

Address for reprints: Jeffrey B. Rubins, MD, Puimonary (111N), VA Medical Center, One Veterans Dr, Minneapolis, MN 55417.

$12 / 1 / 92144$

412 serum CEA assays have low sensitivity and specificity for diagnosing lung cancers, ${ }^{1}$ several reports have indicated that elevated preoperative CEA levels are associated with more advanced disease and with very poor survival after presumptively curative surgical resection. ${ }^{2-6}$

Despite this evidence, the preoperative measurement of serum CEA has not been incorporated into the staging evaluation of patients considered for resection of lung cancer. Because assay of CEA is an inexpensive, readily available preoperative serum test that may correlate with aspects of tumor biology not measured by conventional staging modalities, we sought to re-examine in our clinical practice whether preoperative measurement of serum CEA added useful prognostic data to the current preoperative staging algorithm including computed tomography (CT), bronchoscopy, and mediastinoscopy. In addition, we attempted to characterize the biologic plausibility of CEA as a predictive tool by correlating elevations in serum CEA with tumor size, histologic type, and stage.

\section{Patients and methods}

Selection of patients. From July 1991 through Decem ber 1992, 142 consecutive patients were evaluated by the Pulmonary Medicine Scrvice at the Minneapolis Veterans Affairs Medical Center for lung cancer suspected on the 
basis of abnormal chest radiographs or uncxplained hemoptysis. Of these, 130 patients gave informed consent to phlebotomy of a single blood sample for determination of serum CEA before bronchoscopy, which was the first invasive study performed in all patients. This study was approved by the Institutional Review Board on Human Studies at the Minneapolis Veterans Affairs Medical Center.

In all 130 patients, the disease was staged by history, physical examination, laboratory tests including liver function and serum calcium, CT from the chest through the adrenal glands, and bronchoscopy. Patients in whom advanced disease was suspected on the basis of systemic symptoms (especially weight loss), abnormal laboratory tests (especially elevations in levels of alkaline phosphatase or calcium), or who had larger or more central tumors had additional radiographic studies including CT of the head and abdomen and radionuclide bone scans. Solitary lesions suggestive of metastatic spread of cancer from distant sites were evaluated by CT-guided needle biopsy. All patients with potentially resectable lung cancer underwent exploration by mediastinoscopy and intraoperative bronchoscopy before thoracotomy. Contralateral lymph nodes were sampled during mediastinoscopy, and mediastinal lymph node dissection was performed for pneumonectomy and lobectomy cases. Thoracic surgeons and patients were kept blinded to the results of CEA determinations throughout the study. All thoracotomies were performed within 4 weeks of the serum CEA measurements.

Patient demographic data and pathology reports were obtained from the hospital computer database. Date of last contact or date of death was obtained by scanning the hospital computer database and tumor registry for admissions, outpatient visits, and dates of death. For patients who could not be clearly identified as alive (outpatient or inpatient visit within the past 2 months) or dead from the hospital computer records, the Veterans Administration Beneficiary Identification and Records Locator System database was used to ascertain dates of death.

CEA assay. Blood was allowed to clot, and serum was scparated by centrifugation and frozen at $-20^{\circ} \mathrm{C}$ until assayed. Serum CEA was measured by means of the Tandem solid-phase 2-site radioimmunoassay (Hybritech Inc, San Diego, Calif). The detection limit of the assay was $1 \mathrm{ng} / \mathrm{mL}$, with within-run and between-run coefficients of variation of $5.9 \%$ and $6.9 \%$, respectively. According to the manufacturer, the upper limit of normal for this assay for healthy individuals was $3 \mathrm{ng} / \mathrm{mL}$; serum CEA values excceded $5 \mathrm{ng} / \mathrm{mL}$ and $10 \mathrm{ng} / \mathrm{mL}$, respectively, in $11 \%$ and $7 \%$ of patients with malignant pulmonary disease.

Statistical analysis. The following analytic approach was taken to assess whether preopcrative CEA levels add useful prognostic information about length of survival beyond that supplicd by the standard prognostic variables of pathologic stage, tumor size, histologic type, and patient age at the time of operation. This analysis included only those patients who underwent surgical resection of their tumor $(n=50)$. Pathologic stage was dichotomized into stage 3 or higher versus stages 1 and 2. Histologic type was categorized as adenocarcinoma, squamous cell carcinoma, or other. The date of operation was included
Table I. Estimated probability of 3-year survival for stage 1 and 2 squamous cell and adenocarcinoma by preoperative $C E A$ level

\begin{tabular}{ccc}
\hline $\begin{array}{c}\text { Serum CEA } \\
(\mathrm{ng} / \mathrm{ml})\end{array}$ & $\begin{array}{c}\text { Squamous cell } \\
\text { carcinoma }\end{array}$ & Adenocarcinoma* \\
\hline 1 & $0.58(0.41,0.83)$ & $0.46(0.22,0.94)$ \\
4 & $0.50(0.32,0.78)$ & $0.37(0.16,0.86)$ \\
7 & $0.41(0.22,0.78)$ & $0.28(0.09,0.81)$ \\
10 & $0.32(0.13,0.79)$ & $0.19(0.05,0.81)$ \\
14 & $0.20(0.04,0.97)$ & $0.10(0.01,0.99)$ \\
\hline
\end{tabular}

${ }^{*}$ Numbers in parentheses are $95 \%$ confidence intervals.

in the models to control for any possible bias that would be introduced as a result of the differing lengths of time at risk. Survival was calculated from the date of operation to the date of death or last contact. A Cox proportional hazards model was fitted to the data incorporating the covariates (stage, tumor size, histologic type, age, and date of operation) other than CEA. CEA was then added to the model, after diagnostics examining plots of residuals and tests for power transformations indicated that the only useful transformation was rounding the CEA level to the nearest integer (CEA values $>25$ were coded as 25 ). The likelihood ratio test was used to compare the difference in the two models (with and without CEA). Risk ratios and $95 \%$ confidence intervals were derived from the model. Interaction terms between CEA and histologic typc, size, and stage were subsequently introduced into the model but were not significant and are not presented here. From the model we also derived estimates of the effect of various CEA levels on 3-year survival for patients with stage 1 and 2 squamous cell carcinoma and adenocarcinoma separately, using the observed mean value for the other covariates.

To study the biologic plausibility of CEA as a prognostic indicator in lung cancer, we investigated univariate associations between CEA and histologic type, surgical stage, tumor size, and liver function tests. Because of the skewed distribution of serum CEA concentrations, median values are presented and associations of serum CEA concentrations with histologic type and TNM stage were analyzed by Kruskal-Wallis 1-way analysis of variance with post hoc comparisons by Wilcoxon rank sum tests. Associations between serum CEA concentrations and tumor size were by Pearson bivariate correlation. Analyses were performed by means of SPSS for Windows (release 6.1, Chicago, III) and SAS (release 6.11, SAS Inc, Cary, NC). All $P$ values are two-tailed.

\section{Results}

Between July 1991 and December 1992, 130 of 142 consecutive patients evaluated for suspected lung cancer consented to have serum CEA concentrations measured before diagnostic procedures. In 111 (109 male, 2 female) of these 130 patients, a diagnosis of malignant disease was made by bronchoscopy, needle biopsy, mediastinoscopy, or resection. Sixteen patients were considered not to be 
Table II. Association of serum CEA and histologic type

\begin{tabular}{|c|c|c|c|c|}
\hline \multirow[b]{2}{*}{ Histology } & \multicolumn{2}{|c|}{ Resected cases } & \multicolumn{2}{|c|}{ Total malignant cases } \\
\hline & No. & $\begin{array}{c}C E A: \\
\text { median (range) }\end{array}$ & No. & $\begin{array}{c}C E A: \\
\text { median (range) }\end{array}$ \\
\hline Adenocarcinoma & 21 & $6.1(1.3-910.0)$ & 38 & $7.3 \dagger(1.3-910.0)$ \\
\hline Squamous cell & 22 & $3.9(0-14.6)$ & 39 & $3.7(0-16.1)$ \\
\hline Large cell & 4 & $4.1(1.3-6.9)$ & 15 & $2.9(1.3-6.9)$ \\
\hline Small cel] & 1 & 6 & 14 & $6.4+(2.2-462.0)$ \\
\hline Other* & 2 & $1.3(0.1-2.5)$ & 5 & $2.3(0.1-4.0)$ \\
\hline Total & 50 & & 111 & \\
\hline
\end{tabular}

*Solitary metastatic carcinoma and lymphoma.

$\uparrow P<.01$ compared with squamous cell and large cell carcinoma.

surgical candidates because of preoperative histologic studies showing small cell (13 patients), lymphoma ( 2 patients), or metastatic adenocarcinoma (1 patient). An additional 43 patients had advanced non-small cell lung cancer determined by the staging methods described under Patients and methods (unresectable stage 3A, 2 patients; stage 3B, 23 patients; stage 4, 18 patients). Two additional patients were precluded from surgery because of severe lung disease. Thus 50 of 130 study patients with suspected malignancy had apparently limited lung cancer after a full staging evaluation and were taken to thoracotomy for resection. These 50 resections included 8 pneumonectomies, 39 lobectomies, and 3 wedge resections. Resection margins were histologically free of tumor in all cases. All 3 patients undergoing wedge resections (because of limited cardiopulmonary reserve) had $\mathrm{T} 1$ lesions with no evidence of nodal disease.

In a multivariate analysis of the 50 resected cases, we evaluated the utility of preoperative CEA as a predictor of long-term survival, as described under Patients and methods. The likelihood ratio test comparing the Cox proportional hazards model, first without and then with CEA added as a covariate to the model, indicated that CEA was a significant predictor of survival independent of patient age, pathologic stage, histologic type, and tumor size $(P=.0357)$. The model-estimated risk ratio for CEA level is 1.086 (95\% CI: $1.009,1.169)$, indicating that for each increase of CEA of $1 \mathrm{ng} / \mathrm{mL}$, the hazard (risk) of death increases by $8.6 \%$. Estimated probabilities of 3-year survival by CEA level for stage 1 and 2 adenocarcinoma and squamous cell carcinoma are shown in Table I. For example, the estimated probability of surviving 3 years after surgery for patients with stage 1 or 2 squamous cell carcinoma is approximately $60 \%$ for those with preoperative CEA levels less than $2 \mathrm{ng} / \mathrm{mL}$, but only $40 \%$ for those with CEA levels of $7 \mathrm{ng} / \mathrm{mL}$, and $20 \%$ for those with CEA levels of $14 \mathrm{ng} / \mathrm{mL}$. For patients with stage 1 or 2 adenocarcinoma, the 3-year survival probabilities for these three levels of CEA are approximately $45 \%, 30 \%$, and $10 \%$, respectively. However, the confidence intervals around these survival probability estimates are wide because of the relatively small number of cases available for analysis.

To understand the biologic plausibility of CEA as a predictive tool, we correlated elevations in serum CEA with histologic type, tumor size, and stage. For the total 111 malignant cases, median preoperative serum CEA concentrations were significantly higher for adenocarcinoma and small cell carcinoma than for large cell or squamous cell carcinoma (Table II). For the 50 resected cancers, the association between median serum CEA concentrations and histologic types was not statistically significant, likely because of the small numbers of cases. Because serum CEA concentrations can be elevated by a combination of increased numbers or synthetic rates of malignant cells and decreased clearance through the liver, we hypothesized that serum CEA in patients with lung cancer might be correlated with measures of tumor mass or with hepatic function. However, no significant association between tumor size and serum CEA concentration was identified by Pearson bivariate correlation. Similarly, median serum CEA concentrations were not significantly associated with TNM stage for either the total 111 malignant cases or for the 50 resected cases (Table III). With regard to hepatic clearance, serum CEA concentrations did not correlate with other metabolic tests of liver function, including serum aspartate aminotransferase, bilirubin, alkaline phosphatase, or albumin (not shown).

\section{Discussion}

When applied to a consecutive series of patients evaluated for suspected lung cancer, preoperative serum CEA provided additional prognostic information independent of patient age, pathologic stage, histologic type, and tumor size. Despite the small numbers of cases in our study, elevated preoperative serum CEA was associated with a clinically and statistically significant reduction in survival after intended curative resection. Thus, despite current advanced imaging modalities and diagnostic procedures for preoperative staging of lung cancer, our data support previous studies that have uni- 
formly indicated a role for preoperative serum CEA as an adjunct to conventional modalities for staging lung cancer. ${ }^{4-10}$

The biologic basis for the association between serum CEA and survival in lung cancer remains obscure. Serum CEA is related to tumor histologic type. We found significantly higher serum CEA concentrations in adenocarcinomas and small cell carcinomas than in large cell and squamous cell carcinomas, in agreement with previous reports. $^{7,8,10,11}$ Interestingly, tumor production of CEA measured by immunocytochemical staining or assay of tumor cytosolic concentrations does not correlate with serum CEA concentrations and does not predict survival in lung cancer, even when simultaneously measured serum CEA levels do. ${ }^{2,9,12}$ In addition to the weak relationship between cellular production of CEA and serum levels, we and others found no significant association with primary tumor size and serum CEA concentrations. ${ }^{4,5}$ Although serum CEA may reflect production by tumor at other sites, we found no significant association between TNM stage and serum CEA concentrations in 130 cases, in agreement with other studies. ${ }^{4,6,7}$ In addition, one autopsy study of patients with lung cancer concluded that serum CEA concentrations are not related to the number of organs involved or the sites of metastatic cancer. ${ }^{5}$ Serum CEA is cleared primarily by the liver and is thought to be elevated in colorectal carcinoma in cases of liver metastases. However, we found no association between liver function tests and serum CEA concentrations. Given the size of our study, we cannot definitively exclude a contribution of some of these factors to net serum CEA concentrations. At present, we can only conclude from our study and from published investigations that the exact mechanism of elevated serum CEA concentrations in lung cancers remains unclear.

In summary, serum CEA has all of the properties desired for a biologic measure to be used as a prognostic indicator in the clinical evaluation of lung cancer. It is objective, reproducible, and can be evaluated before the operation. Although a number of other serum tumor markers, including tissue polypeptide antigen and CA-125, appear to also have prognostic utility in the preoperative evaluation of lung cancer, ${ }^{9}$ currently serum CEA is the only one of these that is inexpensive and routinely available. Serum CEA also has distinct advantages over the variety of other tumor markers that are being investigated to better understand lung cancer
Table III. Association of serum CEA with clinical or surgical stage

\begin{tabular}{|c|c|c|c|c|}
\hline \multirow[b]{2}{*}{ Stage } & \multicolumn{2}{|c|}{ Resected cases } & \multicolumn{2}{|c|}{ Total malignant cases } \\
\hline & No. & $\begin{array}{c}C E A: \\
\text { median (range) }\end{array}$ & No. & $\begin{array}{c}C E A: \\
\text { median (range) }\end{array}$ \\
\hline 1 & 28 & $3.2(0-109.0)$ & 28 & $3.2(0-109.0)$ \\
\hline 2 & 7 & $4.2(1.3-11.9)$ & 7 & $4.2(1.3-11.9)$ \\
\hline $3 A$ & 10 & $5.4(2.5-910.0)$ & 12 & $5.2(1.7-910.0)$ \\
\hline $3 B$ & 5 & $6.2(2-14.6)$ & 28 & $3.6(1.8-366.0)$ \\
\hline 4 & 0 & 0 & 18 & $4.7(0.7-35.5)$ \\
\hline Unstaged $^{*}$ & & & 18 & $3.8(0.8-462.0)$ \\
\hline Total & 50 & & 111 & \\
\hline
\end{tabular}

*Cases of small cell, lymphoma, or metastatic cancer.

biology. Markers of histologic features, such as tumor grade, mitotic index, and vascular invasion, and immunocytochemical assays of oncogene products such as p53, ras, and others, ${ }^{13}$ furnish at most semiquantitative information, are subject to interobserver variation, and require resection of a finite volume of tumor for studies to be accomplished. Direct genetic studies, such as DNA sequencing and polymerase chain reaction single-stranded conformational polymorphism, are presently available only as research tools and have the same limitations.

Taking together our data and previously published studies, we conclude that routine preoperative serum CEA measurements are an important adjunct to conventional anatomic tumor staging algorithms, particularly for patients with adenocarcinoma. Marked elevations of serum CEA are indications for full and careful staging despite apparent localized disease on chest radiographs and $\mathrm{CT}$. In such patients, especially those who are marginal surgical candidates due to underlying illnesses, the poor prognosis implied by the test may be factored into decisions regarding surgical resection. Further studies are required to determine whether immediate or delayed postoperative adjuvant treatment might improve the outcome for these patients. Although such therapies have not had conclusive benefit for patients with non-small cell lung cancer, the use of serum tumor markers such as CEA may be a superior means of identifying such patients for these adjuvant therapies.

We thank Ms Patti Baribeau, Minneapolis Veterans Affairs Tumor Registry, and Ms Mary Macauley, Minneapolis Veterans Affairs Department of Thoracic Surgery, for assistance in obtaining patient demographic data, pathology reports, and outcomes. We thank David Nel- 
son, PhD, for invaluable assistance in statistical data analysis.

\section{REFERENCES}

1. NIH. Carcinoembryonic antigen: its role as a marker in the management of cancer. BMJ 1981;282:373-5.

2. Ford CHJ, Stokes HJ, Newman CE. Carcinoembryonic antigen and prognosis after radical surgery for lung cancer: immunocytochemical localization and serum levels. $\mathrm{Br} \mathbf{J}$ Cancer 1981;44:145-53.

3. Dent PB, McCulloch PB, Wesley-James O, MacLaren R, Muirhead W, Dunnett CW. Measurement of carcinoembryonic antigen in patients with bronchogenic carcinoma. Cancer 1978;42;1484-91.

4. Stokes TC, Stevens JFS, Long P, Lockey E, Miller AL. Preoperative carcinoembryonic antigen and survival after resection of lung cancer. $\mathrm{Br} \mathbf{J}$ Dis Chest 1980;74:390-4.

5. Vincent RG, Chu TM, Fergen TB, Ostrander M. Carcinoembryonic antigen in 228 patients with carcinoma of the lung. Cancer 1975;36:2069-76.

6. Concannon JP, Dalbow MH, Hodgson SE, et al. Prognostic value of preoperative carcinoembryonic antigen (CEA) plasma levels in patients with bronchogenic carcinoma. Cancer 1978:42;1477-83.

7. Niklinkski J, Furman M, Laudanski J, Kozlowski M. Prog- nostic value of pretreatment CEA, SCC-Ag and CA 19-9 levels in sera of patients with non-small cell lung cancer. Eur J Cancer Prevent 1992;1:401-6.

8. Niklinkski J, Furman M, Laudanski J, Palynyczko Z, Welk M. Evaluation of carcinoembryonic antigen (CEA) and braintype creatine kinase (CK-BB) in serum from patients with carcinoma of the lung. Neoplasma 1991;38:129-35.

9. Diez M, Torres A, Maestro ML, et al. Prediction of survival and recurrence by serum and cytosolic levels of CEA, CA125 and SCC antigens in resectable non-small-cell lung cancer. Br J Cancer 1996;73:1248-54

10. Icard P, Regnard J, Essomba A, Panebianco V, Magdeleinat P, Levasseur P. Preoperative carcinoembryonic antigen level as a prognostic indicator in resected primary lung cancer. Ann Thorac Surg 1994;58:811-14.

11. Ratto GB, Mereu C, Rovida S. Multiple tumour markers for diagnosis, management and follow-up of potentially resectable lung cancer. Panminerva Med 1993;35:186-92.

12. Graziano SL, Tatum AH, Newman NB, et al. The prognostic significance of neuroendocrine markers and carcinoembryonic antigen in patients with resected stage I and II nonsmall cell lung cancer. Cancer Res 1994;54:2908-13.

13. Greatens TM, Niehans GA, Rubins JB, et al. Do molecular markers predict survival in non-small cell lung cancer? Am J Respir Crit Care Med 1998;157:1093-7.

\section{Availability of Journal back issues}

As a scrvice to our subscribers, copies of back issues of The Journal of Thoracic and Cardiovascular Surgery for the preceding 5 years are maintained and are available for purchase from Mosby at a cost of $\$ 16.00$ per issue until inventory is depleted. The following quantity discounts are available: $25 \%$ off on quantities of 12 to 23 , and one third off on quantities of 24 or more. Please write to Mosby, Inc, Subscription Services, 11830 Westline Industrial Drive, St Louis, MO 63146-3318, or call 800-453-4351 or 314-453-4.351 for information on availability of particular issues. If unavailable from the publisher, photocopies of complete issues may be purchased from UMI, $300 \mathrm{~N}$ Zeeb Rd, Ann Arbor, MI 48106, 313-761-4700. 